\title{
Hysteretic jumps in the response of layered superconductors to electromagnetic fields
}

\author{
V.A. Yampol'skii, ${ }^{1,2}$ T.M. Slipchenko, ${ }^{2,3}$ Z.A. Mayzelis, ${ }^{4,3}$ \\ D.V. Kadygrob, ${ }^{3}$ S.S. Apostolov, ${ }^{2,3}$ S.E. Savel'ev, ${ }^{1,5}$ and Franco Nori ${ }^{1,6}$ \\ ${ }^{1}$ Advanced Science Institute, The Institute of Physical and Chemical Research (RIKEN), \\ Wako-shi, Saitama, 351-0198, Japan \\ ${ }^{2}$ A.Ya. Usikov Institute for Radiophysics and Electronics \\ Ukrainian Academy of Sciences, 61085 Kharkov, Ukraine \\ ${ }^{3}$ V.N. Karazin Kharkov National University, 61077 Kharkov, Ukraine \\ 4 A.Ya. Usikov Institute for Radiophysics and Electronics \\ Ukrainian Academy of Sciences, 61085 Kharkov, Ukraine \\ ${ }^{5}$ Department of Physics, Loughborough University, Loughborough LE11 3TU, UK \\ ${ }^{6}$ Department of Physics, Center for Theoretical Physics, \\ Applied Physics Program, Center for the Study of Complex Systems, \\ University of Michigan, Ann Arbor, MI 48109-1040, USA
}

(Dated: November 23, 2018)

\begin{abstract}
We consider here a layered superconductor subject to an externally applied moderately-strong electromagnetic field. We predict hysteretic jumps in the dependence of the surface reactance of the superconductor on the amplitude $H_{0}$ of the incident electromagnetic wave. This very unusual nonlinear phenomenon can be observed in thin superconducting slabs at not very strong ac amplitudes, if the frequency of the irradiating field is close to the Josephson plasma frequency. Using the set of coupled sine-Gordon equations, we derive the expression for the phase shift $\chi$ of the reflected wave and obtain the conditions for the appearance of hysteresis in the $\chi\left(H_{0}\right)$-dependence.
\end{abstract}

PACS numbers: $74.78 . F k 74.50 .+\mathrm{r}$ 


\section{INTRODUCTION}

There has been a recent surge of studies of electromagnetic waves (EMWs) propagating in artificially fabricated media (see, e.g., Refs. 1,2,3), including metals with modulated properties $^{4}$, arrays of coupled waveguides ${ }^{5}$, left-hand materials ${ }^{6,7}, \frac{8}{}$, and layered superconductors ${ }^{9}$. The excitation of these waves can produce a large variety of resonance anomalies 10 in the reflectivity, transmissivity, and absorptivity, offering new types of optical nano-devices.

The recent increase of these type of studies is related to nonlinear surface and waveguide EM modes (see, e.g., Refs. 1, 5, 7). In this broad context, a layered superconductor is a medium favoring the propagation of nonlinear ${ }^{11,12,13}$ and surface ${ }^{9}$ waves in the (important for applications 14,15$)$ terahertz $(\mathrm{THz})$ and sub- $\mathrm{THz}$ frequency ranges. Both the existence of surface waves ${ }^{9}$ and the nonlinear effects $11,12,13$ occur due to the gap structure (see, e.g., Ref. 16) of the spectrum of Josephson plasma waves, which was experimentally observed via Josephson plasma resonance ${ }^{17}$. The nonlinearity of Josephson plasma waves with frequency $\omega$ close to the Josephson plasma frequency $\omega_{J}$ becomes important even at small field amplitudes $\propto\left|1-\omega^{2} / \omega_{J}^{2}\right|^{1 / 2}$. In close analogy to nonlinear optics 18 , the nonlinear JPWs exhibit numerous remarkable features ${ }^{11,12,13}$, including the slowing down of light, self-focusing effects, and the pumping of weaker waves by stronger ones. However, the nonlinearity of EMWs in layered superconductors is quite different from optical nonlinearities. This leads one to expect very unusual phenomena in the EMW propagation in this nonlinear media.

In this paper, we predict and analyze theoretically one of such unexpected nonlinear effects in a thin slab of a layered superconductor subject to an externally applied electromagnetic wave. We show that, under specific conditions, the amplitude dependence of the phase $\chi$ of the reflected wave becomes many-valued. This should result in hysteretic jumps of $\chi$ when sweeping the amplitude of the incident wave, a phenomenon which is very unusual for conductors and superconductors.

\section{PROBLEM STATEMENT AND EQUATIONS FOR THE ELECTROMAG- NETIC FIELD}

Consider a slab of a layered superconductor of thickness $d$ (see Fig. 1). The crystallographic ab-plane coincides with the $x y$-plane and the c-axis is along the $z$-axis. The 
interlayer distance $D$ is much smaller than the thickness $d$ of the slab.

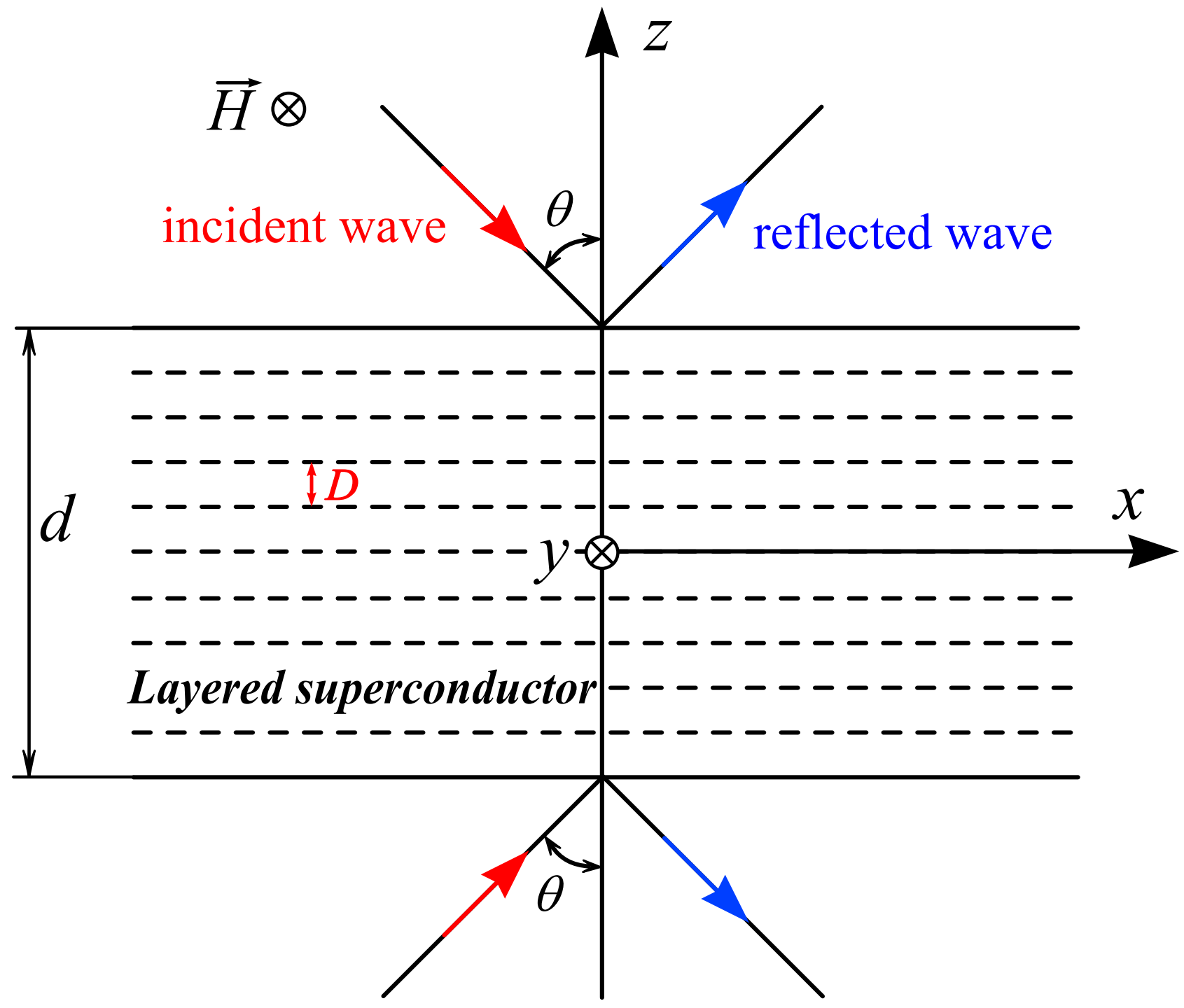

FIG. 1: (Color online) Geometry of the problem. The slab of a layered superconductor is irradiated with a $p$-polarized electromagnetic wave with the magnetic field symmetrical with respect to the middle of the sample.

Let the sample be irradiated by two $p$-polarized (Transverse Magnetic) plane monochromatic electromagnetic waves with the magnetic fields symmetric with respect to the middle of the sample, the plane $z=0$. Therefore, the magnetic $\vec{H}=\{0, H, 0\}$ and electric $\vec{E}=\left\{E_{x}, 0, E_{z}\right\}$ fields satisfy the symmetry conditions,

$$
H(x, z, t)=H(x,-z, t)
$$




$$
E_{x}(x, z, t)=-E_{x}(x,-z, t), E_{z}(x, z, t)=E_{z}(x,-z, t) .
$$

Due to this symmetry, we will only consider the field distribution in the upper half-space $z>0$.

The electromagnetic field in the vacuum, $z>d / 2$, is the sum of the incident and specularly reflected waves. The Maxwell equations give for them,

$$
\begin{aligned}
& H^{V}(x, z, t)=H_{0} \cos \gamma_{-}+H_{r} \cos \left(\gamma_{+}+\chi\right), \\
& E_{x}^{V}(x, z, t)=-\frac{k_{z}}{k}\left(H_{0} \sin \gamma_{-}-H_{r} \sin \left(\gamma_{+}+\chi\right)\right), \\
& \quad \gamma_{-}=k_{x} x-k_{z} z-\omega t, \quad \gamma_{+}=k_{x} x+k_{z} z-k_{z} d-\omega t,
\end{aligned}
$$

with $k_{x}=k \sin \theta, k_{z}=k \cos \theta, k=\omega / c$. Here $\omega$ is the wave frequency, $c$ is the speed of light, and $\theta$ is the angle of incidence. The value of $\chi$ is the phase shift of the reflected wave at the boundary $z=d / 2$ of the slab. As is known, $\chi$ defines the surface reactance $X$ of a sample: when neglecting the dissipation,

$$
X=\frac{4 \pi}{c} \tan \left(\frac{\chi}{2}\right) \cos \theta
$$

Inside a layered superconductor, the electromagnetic field is determined by the interlayer gauge-invariant phase difference $\varphi$ of the order parameter. The spatial distribution of $\varphi(x, z, t)$ obeys the set of coupled sine-Gordon equations (see, e.g., Ref. 19,20). We consider the nonlinear JPWs with $|\varphi| \ll 1$, when the Josephson current $J_{c} \sin \varphi$ can be approximated by $J_{c}\left(\varphi-\varphi^{3} / 6\right)$. We also assume that the gauge-invariant phase difference experiences small changes on the scale $D$ in the $z$-direction, and thus we can use the continuum approach. In the continuum limit, the coupled sine-Gordon equation has the form,

$$
\left(1-\lambda_{a b}^{2} \frac{\partial^{2}}{\partial z^{2}}\right)\left(\frac{1}{\omega_{J}^{2}} \frac{\partial^{2} \varphi}{\partial t^{2}}+\varphi-\frac{\varphi^{3}}{6}\right)-\lambda_{c}^{2} \frac{\partial^{2} \varphi}{\partial x^{2}}=0 .
$$

Here $\lambda_{a b}$ and $\lambda_{c}=c / \omega_{J} \varepsilon^{1 / 2}$ are the London penetration depths across and along layers, respectively, and $\omega_{J}=\left(8 \pi e D J_{c} / \hbar \varepsilon\right)^{1 / 2}$ is the Josephson plasma frequency. The latter is determined by the maximum Josephson current $J_{c}$, the interlayer dielectric constant $\varepsilon$, and the interlayer spacing $D$. The spatial variations in the $z$-direction of the fields inside the very thin superconducting layers are neglected. Here we also omit the dissipation terms related to the quasiparticle conductivity. They are controlled by the sample temperature $T$ and can be reduced to negligibly small values. Moreover, in Eq. (3), we neglect the term 
with the capacitive coupling, for waves with sufficiently high $k_{x}=k \sin \theta \sim \omega / c \gg \beta / \lambda_{c}$. Here $\beta=R_{D}^{2} \varepsilon / s D \ll 1$ is the prefactor of the capacitive coupling 21 , $R_{D}$ is the Debye length for a charge in a superconductor, and $s$ is the thickness of the superconducting layers.

The magnetic and electric fields in a layered superconductor are related to the gaugeinvariant phase difference as

$$
\begin{aligned}
& \frac{\partial H^{S}}{\partial x}=\frac{\mathcal{H}_{0}}{\lambda_{c}}\left(\frac{1}{\omega_{J}^{2}} \frac{\partial^{2} \varphi}{\partial t^{2}}+\varphi-\frac{\varphi^{3}}{6}\right), \\
& E_{x}^{S}=-\frac{\lambda_{a b}^{2}}{c} \frac{\partial^{2} H^{S}}{\partial z \partial t}, \quad \mathcal{H}_{0}=\frac{\Phi_{0}}{2 \pi D \lambda_{c}},
\end{aligned}
$$

where $\Phi_{0}=\pi c \hbar / e$ is the flux quantum and $e$ is the elementary charge.

As was shown in Ref. 13, the nonlinearity in Eq. (3) can play a crucial role in the wave propagation for frequencies close to $\omega_{J}$, i.e., for $\left|1-\Omega^{2}\right| \equiv\left|1-\omega^{2} / \omega_{J}^{2}\right| \ll 1$. Indeed, if $\varphi \sim\left|1-\Omega^{2}\right| \ll 1$, the cubic term $\varphi^{3}$ in Eq. (3) is of the same order as the linear term $\omega_{J}^{-2} \partial^{2} \varphi / \partial t^{2}+\varphi$.

We consider the frequency range below the Josephson plasma frequency, $\Omega<1$, and seek a solution of Eq. (3) of the form,

$$
\begin{aligned}
\varphi(x, z, t) & =a(z)\left(1-\Omega^{2}\right)^{1 / 2} \sin \left(\gamma_{0}+\alpha\right), \\
\gamma_{0} & =k_{x} x-k_{z} d / 2-\omega t
\end{aligned}
$$

keeping only the first harmonics in $\left(k_{x} x-\omega t\right)$.

Substituting $\varphi$ in Eq. (5) into Eq. (4), one obtains

$$
\begin{aligned}
& H^{S}(x, \zeta, t)=-\mathcal{H}_{0} \frac{\left(1-\Omega^{2}\right)}{\kappa} h(\zeta) \cos \left(\gamma_{0}+\alpha\right), \\
& E_{x}^{S}(x, \zeta, t)=\mathcal{H}_{0} \frac{\left(1-\Omega^{2}\right)}{\kappa} P h^{\prime}(\zeta) \sin \left(\gamma_{0}+\alpha\right) .
\end{aligned}
$$

Here we introduce the dimensionless variables,

$$
h(\zeta)=a(\zeta)-\frac{a^{3}(\zeta)}{8}, \quad \zeta=\frac{\kappa z}{\lambda_{a b}}
$$

and parameters,

$$
P=\frac{\lambda_{a b}}{\lambda_{c}} \frac{\kappa}{\sqrt{\varepsilon}}, \quad \kappa=\frac{\lambda_{c} k_{x}}{\left(1-\Omega^{2}\right)^{1 / 2}},
$$

and the prime denotes $d / d \zeta$.

Equations (3) and (15) yield the ordinary second-order differential equation for $a(z)$ :

$$
\left[1-\kappa^{2} \frac{d^{2}}{d \xi^{2}}\right]\left(a-\frac{a^{3}}{8}\right)+\kappa^{2} a=0 .
$$


Further, we also assume $\kappa \gg 1$, which is valid for not very small incident angles $\theta$. In this case, integrating Eq. (9) with the symmetry condition,

$$
a^{\prime}(0)=0
$$

we obtain

$$
\frac{3}{4}\left(a^{\prime}\right)^{2}=\left(\frac{8-3 a_{0}^{2}}{8-3 a^{2}}\right)^{2}-1,
$$

where $a_{0}=a(0)$. The solution of Eq. (10) can be written in the implicit form,

$$
\zeta=\sqrt{\frac{3}{4}} \int_{a_{0}}^{a(\zeta)} d a \frac{8-3 a^{2}}{\sqrt{\left(8-3 a_{0}^{2}\right)^{2}-\left(8-3 a^{2}\right)^{2}}}
$$

The phase diagram, i.e., the set of $a^{\prime}(a)$ curves for different values of the constant $a_{0}$, is shown in Fig. 2, Solid circles mark the sample boundaries, while open circles indicate the middle of the slab. Arrows show the direction of motion along the phase trajectories when increasing the coordinate $\zeta$.

Thus the electromagnetic fields in the vacuum and in the superconducting slab are determined by Eqs. (2) and (6), respectively. The latter equations contain the function $a(\zeta)$ given by Eq. (11).

\section{PHASE SHIFT OF THE REFLECTED WAVE}

Now we find the relationship between the phase shift $\chi$ of the reflected wave and the amplitude $H_{0}$ of the incident wave. With this purpose, we join the tangential components of the electric and magnetic fields in the vacuum and in the superconducting slab, at the interface $z=d / 2$. Thus, separating terms with $\sin \left(k_{x} x-\omega t\right)$ and $\cos \left(k_{x} x-\omega t\right)$, we derive four equations for $H_{r}, H(d / 2), \chi$, and $\alpha$ :

$$
\begin{aligned}
-h_{0}+h_{r} \cos \chi & =P h^{\prime}(\delta) \sin \alpha, \\
-h_{0}-h_{r} \cos \chi & =h(\delta) \cos \alpha, \\
-h_{r} \sin \chi & =P h^{\prime}(\delta) \cos \alpha, \\
-h_{r} \sin \chi & =h(\delta) \sin \alpha .
\end{aligned}
$$

Here

$$
h_{0}=\frac{H_{0}}{\mathcal{H}_{0}} \frac{\kappa}{\left(1-\Omega^{2}\right)}, \quad h_{r}=\frac{H_{r}}{\mathcal{H}_{0}} \frac{\kappa}{\left(1-\Omega^{2}\right)} ;
$$




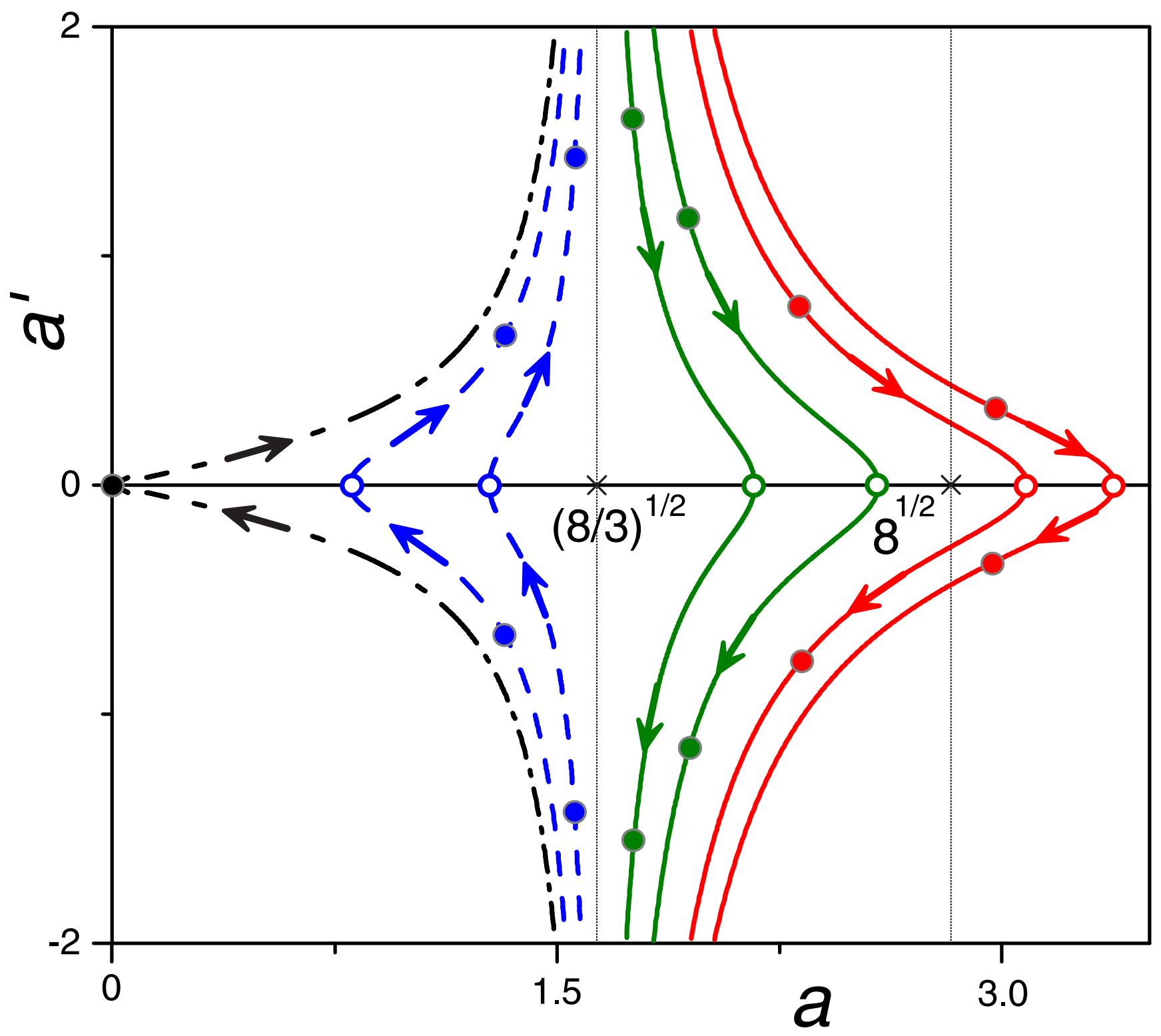

FIG. 2: (Color online) The phase diagram $a^{\prime}(a)$ (for $a>0$ ). Recall that $a$ is the amplitude of the gauge-invariant phase $\varphi$ (see Eq. (5) ), while $a^{\prime}$ is its derivative with respect to the dimensionless coordinate $\zeta=\kappa z / \lambda_{a b}$. The paths along the arrows on the phase trajectories between solid circles correspond to the change of the coordinate $z$ inside the sample, from $z=-d / 2$ to $z=d / 2$. Open circles correspond to the middle of the slab $(z=0)$.

$\delta=\kappa d / 2 \lambda_{a b}$ is the value of $\zeta$ at the interface $z=d / 2$. Note that, without any loss of generality, we can assume the value $h(\delta)$ of the total magnetic field at the sample surface to be positive, $h(\delta)>0$. A negative $h(\delta)$ correspond to replacing $\chi \rightarrow \chi+\pi$ in Eqs. (12). 
Excluding $h_{r}$ and $\alpha$ from Eqs. (12), we find

$$
\begin{aligned}
h_{0} & =\frac{1}{2} \sqrt{h^{2}(\delta)+\left(P h^{\prime}(\delta)\right)^{2}} \\
\chi & =2 \arctan \left(\frac{P h^{\prime}(\delta)}{h(\delta)}\right) .
\end{aligned}
$$

Equations (13), (14), together with Eqs. (7) and (11), give, in an implicit form, the required dependence of the phase shift $\chi$ on the amplitude of the incident wave $H_{0}$.

For further analysis of the amplitude dependence of the phase shift $\chi$, it is very important to take into account the non-single-valued relation between the values $h(\delta)$ and $a(\delta)$. Indeed, Eq. (7) and Fig. 3 show that there exist three values of $a$ that correspond to the same value of $h$, if $0<h<(32 / 27)^{1 / 2}$. Taking into account Eq. (13), we conclude that three different values of the parameter $h^{\prime}(\delta) / h(\delta)$ in Eq. (14) correspond to the same value of $h_{0}$. This results in the appearance of three branches of the dependence $\chi\left(h_{0}\right)$.

Below, for simplicity, we restrict ourselves to the case of small sample thicknesses, when

$$
\delta \ll 1 .
$$

In this case, $\left|a(\zeta)-a_{0}\right| \ll 1$, and Eq. (11) is significantly simplified,

$$
a(\zeta) \simeq a_{0}\left(1+\frac{4 \zeta^{2}}{8-3 a_{0}^{2}}\right),
$$

and one can easily derive the asymptotic equations for all three branches $\chi\left(h_{0}\right)$.

\section{A. Low-amplitude branch $\chi\left(h_{0}\right)$}

First, we discuss the branch of the dependence $\chi\left(h_{0}\right)$ that corresponds to the portion "1-2" of the $h(a)$ curve in Fig. 3. This monotonically increasing branch is shown by the blue curve (with number 1) in the main panel of Fig. 4, and also in inset (a) of Fig. 4. This blue branch is defined within the interval $\left(0, h_{0 \text { cr }}\right)$ of the $h_{0}$ change. The ending point $h_{0 \text { cr }}$ of the branch is described by the expression,

$$
h_{0 \mathrm{cr}} \simeq \sqrt{\frac{8}{27}}+\sqrt{\frac{3}{8}} P^{2} \delta^{2} .
$$

At this point,

$$
\chi\left(h_{0 \text { cr }}\right) \simeq 3 P \delta-\sqrt{\frac{9}{8}} P \delta^{2} .
$$




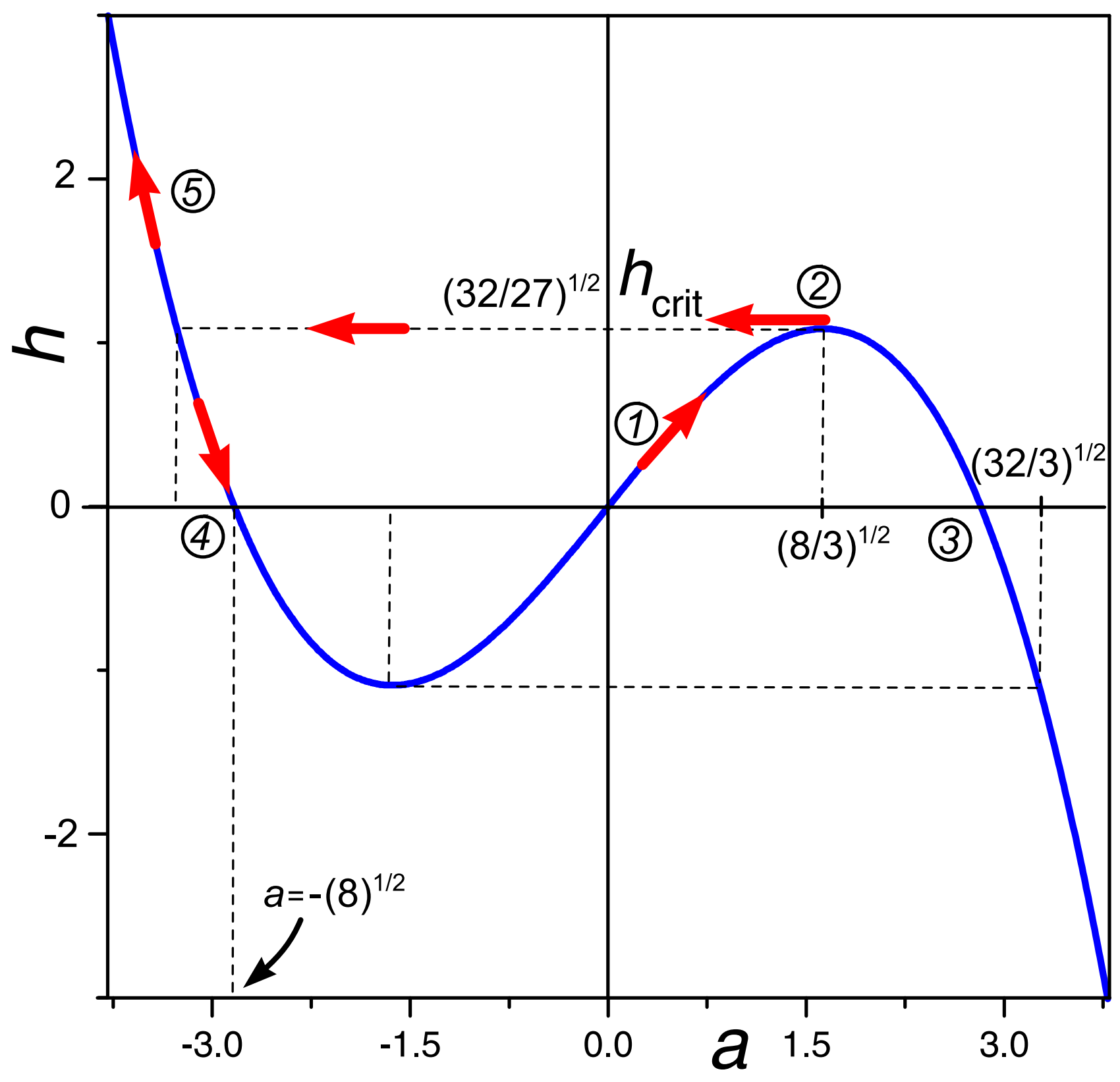

FIG. 3: (Color online) The normalized magnetic field $h$ versus the normalized amplitude $a$ of the gauge-invariant phase $\phi$. This $h(a)$ dependence is described by Eq. (7). Arrows indicate the motion along the $h(a)$ curve when changing the normalized amplitude $h_{0}$ of the incident wave.

The low-amplitude asymptotics of $\chi\left(h_{0}\right)$ dependence is,

$$
\chi \simeq 2 P \delta\left(1+\frac{h_{0}^{2}}{2}\right), \quad h_{0} \ll 1 .
$$




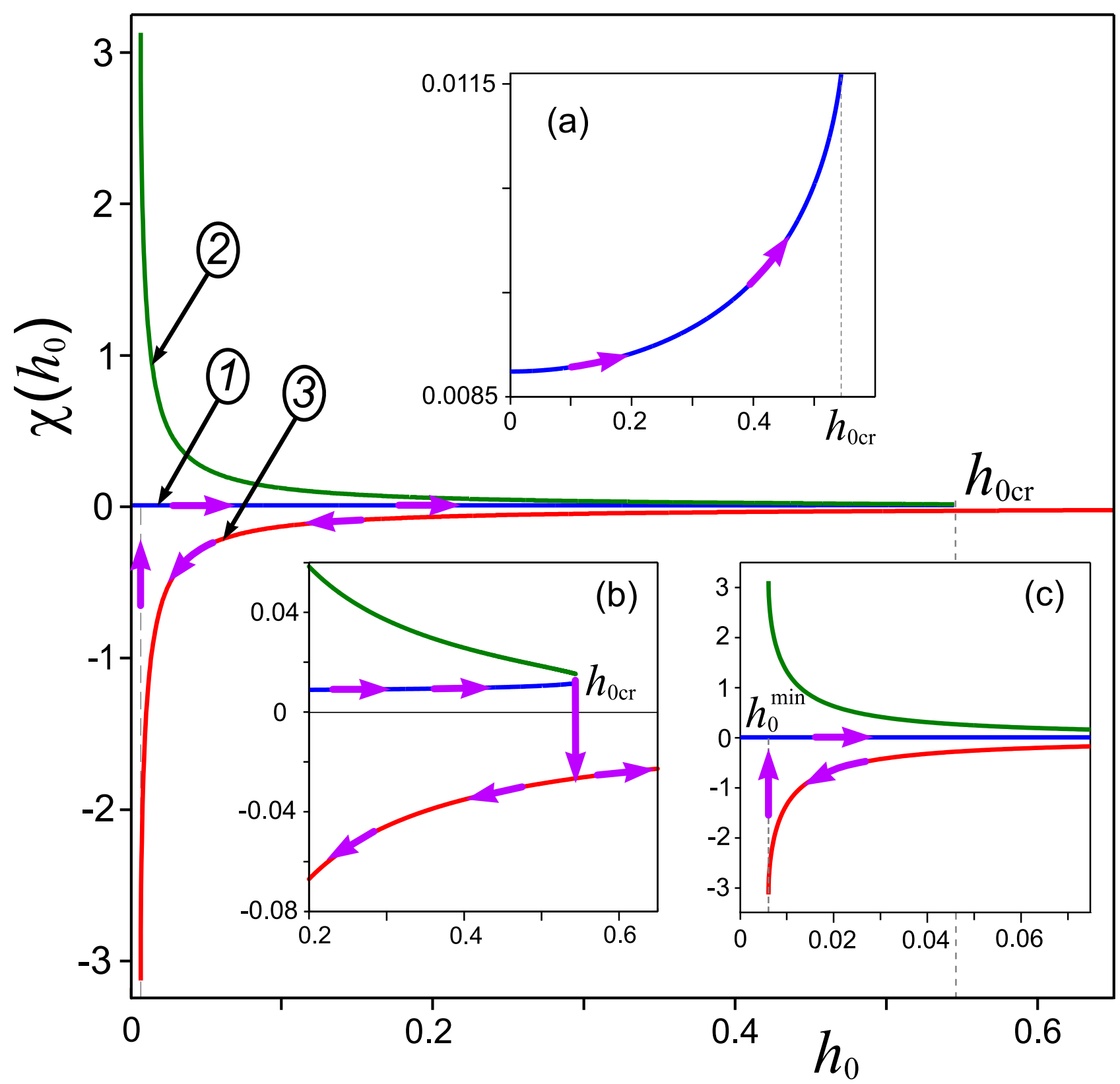

FIG. 4: (Color online) The numerically-obtained dependence of the phase shift $\chi$ of the reflected wave on the dimensionless amplitude $h_{0}$ of the incident electromagnetic wave. The values of the parameters here are: $d / \lambda_{a b}=0.05, \lambda_{c} / \lambda_{a b}=200, \kappa=10$, and $\varepsilon=16$. The insets show magnified portions of the $\chi\left(h_{0}\right)$ plots. The arrows on the curves indicate how the phase shift $\chi$ changes when periodically varying the amplitude $h_{0}$. The first jump in $\chi\left(h_{0}\right)$ at $h_{0}=h_{0 \mathrm{cr}}$ is shown by the downwards vertical arrow in the inset (b). This produces a jump $\Delta \chi \approx-9 P \delta$. The second (reverse) jump at $h_{0}=h_{0}^{\min }$ is shown in the main frame and in the inst (c). Namely, the upward vertical arrow (from $\left.\chi\left(h_{0}\right)\right|_{\text {before jump }}=-\pi$, to $\left.\chi\left(h_{0}\right)\right|_{\text {after jump }} \approx 0$ ) shows the jump in $\chi\left(h_{0}\right)$. 


\section{B. High-amplitude branch $\chi\left(h_{0}\right)$}

The second and third branches of the $\chi\left(h_{0}\right)$ dependence correspond to the portions " $2-3$ " and " $4-5$ ", respectively, of the $h(a)$ curve in Fig. 3 . The second and third branches of $\chi\left(h_{0}\right)$ are shown by the green and red curves (indicated by numbers 2 and 3, respectively) in Fig. 4 . The second branch exists in the interval

$$
h_{0}^{\min }<h_{0}<h_{0 \mathrm{cr}}, \quad h_{0}^{\min } \simeq \sqrt{2} P \delta .
$$

As is seen in Fig. 4, the first and second branches of $\chi\left(h_{0}\right)$ almost meet at the point $h_{0 \mathrm{cr}}$. The third branch is defined for

$$
h_{0}^{\min }<h_{0}<\infty .
$$

Near the minimum value of $h_{0}$, i.e., for $h_{0} \sim h_{0}^{\min }$, the second and third branches have the asymptotics,

$$
\chi\left(h_{0}\right)= \pm\left(\pi-\sqrt{\frac{2 h_{0}^{2}}{\delta^{2}}-4 P^{2}}\right) .
$$

Here, the signs "+" and "-" correspond to the second and third branches, respectively.

The third branch tends to zero for $h_{0} \rightarrow \infty$ following the expression,

$$
\chi\left(h_{0}\right)=-P \delta\left(\frac{4}{h_{0}}\right)^{2 / 3}, \quad h_{0} \gg 1 .
$$

Now we present the parametrically-defined formula for all three branches, which is valid for $h_{0}$ not very close to $h_{0 \text { cr }}$ :

$$
\begin{aligned}
h_{0}\left(a_{0}\right) & =\frac{a_{0}\left|8-a_{0}^{2}\right|}{16}, \\
\chi\left(a_{0}\right) & =\frac{16 P}{8-a_{0}^{2}} \delta .
\end{aligned}
$$

Figure 5 demonstrates the very good agreement of this formula with numerical results.

\section{Hysteresis jumps in the dependence $\chi\left(h_{0}\right)$}

From the analysis shown above, we can now describe the behavior of the phase shift $\chi$ of the reflected wave when periodically changing the amplitude $h_{0}$ of the incident wave.

When increasing $h_{0}$ from zero, the phase shift $\chi\left(h_{0}\right)$ increases monotonically following the first branch in Fig. 4. At the point $h_{0}=h_{0 \text { cr }}$, the first branch comes to the end, and a 


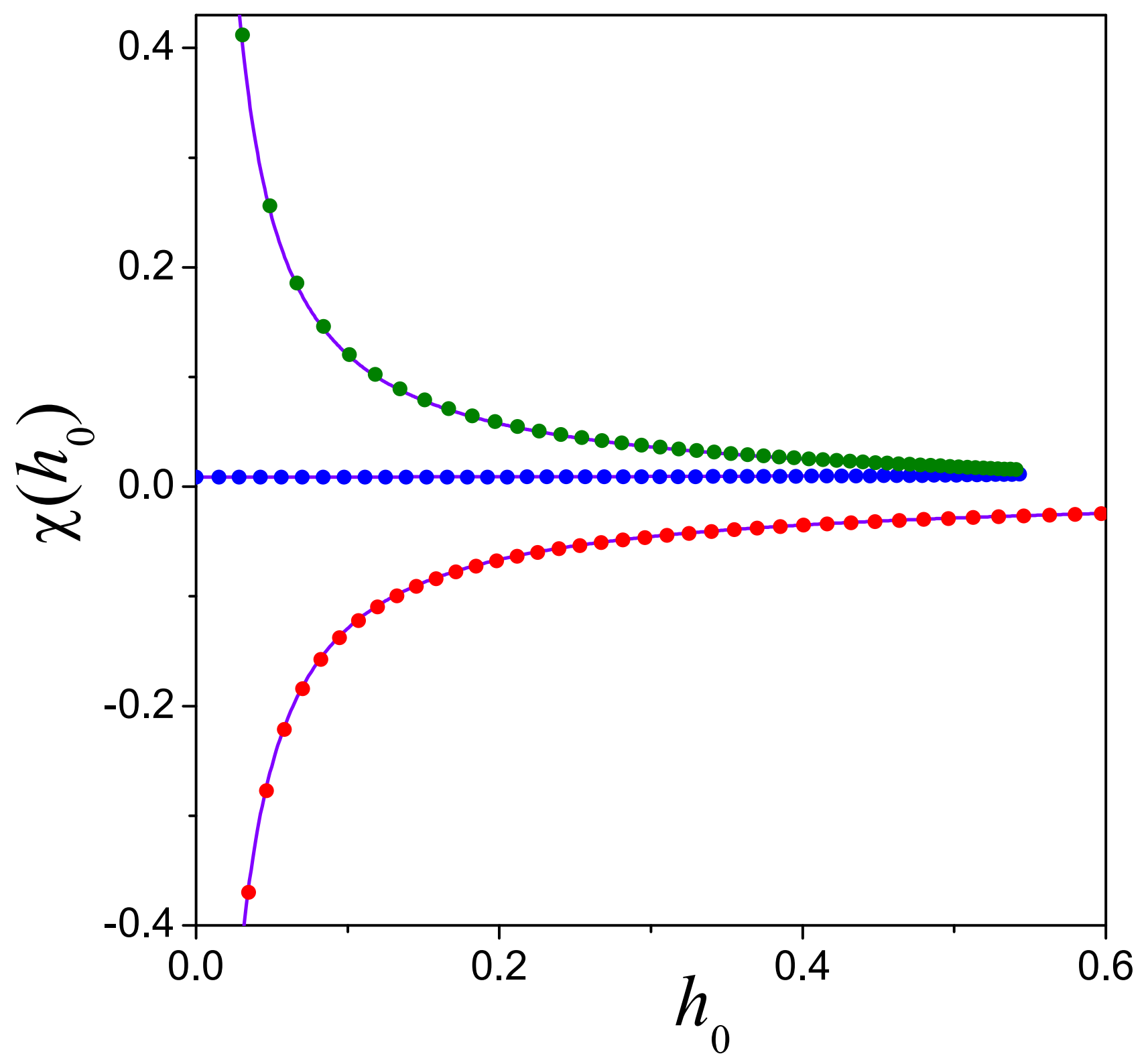

FIG. 5: (Color online) The phase shift $\chi$ of the reflected wave versus the dimensionless amplitude $h_{0}$ of the incident wave, for $d / \lambda_{a b}=0.05, \lambda_{c} / \lambda_{a b}=200, \kappa=10$, and $\varepsilon=16$. The solid curves are the plots of the three branches of the function given by Eqs. (24), while the dots were numerically obtained from Eqs. (7), (11), (13), and (14).

jump to the third branch should occur when further increasing $h_{0}$. The phase shift $\chi\left(h_{0}\right)$ jumps from the value

$$
\chi\left(h_{0 \mathrm{cr}}-0\right) \simeq 3 P \delta
$$

to the final value

$$
\chi\left(h_{0 \mathrm{cr}}+0\right) \simeq-6 P \delta .
$$


Thus, the jump here is

$$
\left.\Delta \chi\right|_{h_{0}=h_{0 \mathrm{cr}}} \approx-9 P \delta .
$$

When decreasing $h_{0}$, the phase shift $\chi\left(h_{0}\right)$ decreases following the third branch, crosses the point $h_{0}=h_{0 \mathrm{cr}}$, and only at the point $h_{0}=h_{0}^{\min }$ it performs a reverse jump to the first branch. Therefore, now

$$
\left.\Delta \chi\right|_{h_{0}=h_{0}^{\min }} \approx \pi
$$

Thus, the hysteretic jumps $\Delta \chi$ of the phase $\chi\left(h_{0}\right)$ of the reflected wave could be observed when periodically changing the amplitude $h_{0}$ of the incident wave.

\section{CONCLUSION}

In this paper, we have predicted and theoretically analyzed unusual phenomenon for conducting media. We have shown that, due to the specific nonlinearity of layered superconductors, hysteretic jumps of the surface reactance could be observed when periodically changing the amplitude $H_{0}$ of the incident wave. A remarkable feature of the predicted phenomenon is the relatively small values of the necessary ac amplitudes $H_{0}$. The hysteretic jumps can be observed even when $\varphi \ll 1$. According to our analysis, the critical amplitude

$$
H_{0 \mathrm{cr}}=h_{0 \mathrm{cr}} \cdot \mathcal{H}_{0} \frac{\left(1-\Omega^{2}\right)}{\kappa} \ll \mathcal{H}_{0} \sim 20 \text { Oe. }
$$

There are two small parameter here, $\left(1-\Omega^{2}\right)$ and $1 / \kappa$. As was shown in Ref. 13, the heating effect is negligible for such ac amplitudes.

The phenomenon discussed here is another exciting example of the numerous unusual effects related to the very specific nonlinearity of layered superconductors.

\section{Acknowledgments}

We gratefully acknowledge partial support from the National Security Agency (NSA), Laboratory of Physical Sciences (LPS), Army Research Office (ARO), National Science Foundation (NSF) grant No. EIA-0130383, JSPS-RFBR 06-02-91200, and Core-to-Core (CTC) program supported by Japan Society for Promotion of Science (JSPS). S.S. acknowl-

edges support from the Ministry of Science, Culture and Sport of Japan via the Grant-in 
Aid for Young Scientists No 18740224, the EPSRC via No. EP/D072581/1, EP/F005482/1, and ESF network-programme "Arrays of Quantum Dots and Josephson Junctions".

1 D.N. Christodoulides, F. Lederer, and Ya. Silberger, Nature (London) 424, 817 (2003).

2 B. Barnes and R. Sambles, Phys. World 19, No 1, 17 (2006); Th. Krauss, Phys. World 19, No $2,32(2006)$.

3 T.W. Ebbesen, C. Genet, and S.I. Bozhevolnyi, Phys. Today 61, No 5, 44 (2008).

4 A.V. Kats, M.L. Nesterov, and A.Yu. Nikitin, Phys. Rev. B 72, 193405 (2005); A.V. Kats and I.S. Spevak, Phys. Rev. B 65, 195406 (2002).

5 S. Suntsov et al., Phys. Rev. Lett. 96, 063901 (2006); J. Hudock et al., Optics Express 13, 7720 (2005).

6 R. Ruppin, J. Phys.: Condens. Matter 13, 1811 (2001); I.V. Shadrivov, A.A. Sukhorukov, and Yu.S. Kivshar, Phys. Rev. E 67, 057602 (2003); A.V. Kats, S. Savel'ev, V.A. Yampol'skii, and F Nori, Phys. Rev. Lett. 98, 073901 (2007).

7 I.V. Shadrivov, A.A. Sukhorukov, Yu.S. Kivshar, A.A. Zharov, A.D. Boardman, P. Egan, Phys. Rev. E 69, 016617 (2004).

8 K. Bliokh et al., Rev. Mod. Phys, in press (2008).

9 S. Savel'ev, V. Yampol'skii, and F. Nori, Phys. Rev. Lett. 95, 187002 (2005); S. Savel'ev, V. Yampol'skii, and F. Nori, Physica C 445-448, 183 (2006).

10 A. Hessel and A.A. Oliner, Appl. Opt. 4, 1275 (1965); V. M. Agranovich and D. L. Mills, Surface Polaritons (Nauka, Moscow, 1985); H. Raether, Surface Plasmons (Springer, New York, 1988); R. Petit, Electromagnetic Theory of Gratings (Springer, Berlin, 1980); W. L. Barnes, A. Dereux, and T. W. Ebbesen, Nature (London) 424, 824 (2003); A. V. Kats and A. Yu. Nikitin, Phys. Rev. B 70, 235412 (2004).

11 S. Savel'ev, A.L. Rakhmanov, V.A. Yampol'skii, and F. Nori, Nat. Phys. 2, 521 (2006).

12 S. Savel'ev, V.A. Yampol'skii, A.L. Rakhmanov, and F. Nori, Phys. Rev. B 75, 184503 (2007).

13 V.A. Yampol'skii, S. Savel'ev, A.L. Rakhmanov, and F. Nori, Phys. Rev. B 78, 024511 (2008).

14 Special Issue of Philosophical Transactions: Mathematical, Physical \& Engineering Science 362, No 1815 (2004).

15 M. Tonouchi, Nat. Phot. 1, 97 (2007). 
16 T. M. Mishonov, Phys. Rev. B 44, 12033 (1991).

17 K. Tamasaku, Y. Nakamura, and S. Uchida, Phys. Rev. Lett. 69, 1455 (1992); O.K.C. Tsui, N.P. Ong, Y. Matsuda, Y.F. Yan, and J.B. Peterson, Phys. Rev. Lett. 73, 724 (1994); Y. Matsuda, M.B. Gaifullin, K. Kumagai, K. Kadowaki, and T. Mochiku, Phys. Rev. Lett. 75, 4512 (1995).

18 D.L. Mills, Nonlinear Optics, (Springer, Berlin, 1998); N. Bloembergen, Nonlinear Optics, (World Scientific, Singapur, 1996); Y.R. Shen, The Principles of Nonlinear Optics, (WileyInterscience, Hoboken, NJ, 2003).

19 A. Barone and G. Paterno, Physics and Applications of the Josephson Effect (Wiley, New York, 1982).

20 S. Sakai, P. Bodin, N.F. Pedersen, J. Appl. Phys. 73, 2411 (1993); S.N. Artemenko and S.V. Remizov, Physica C 362, 200 (2001).

21 T. Koyama and M. Tachiki, Phys. Rev. B 54, 16183 (1996). 